УДК 330.101.541

$10.17213 / 2075-2067-2021-1-203-219$

\title{
МАКРОЭКОНОМИЧЕСКАЯ ПОЛИТИКА: ЭФФЕКТ ПРЕВЫШЕНИЯ СЛОЖНОСТИ
}

\author{
(C) 2021 г. O. C. Cyxapes
}

\section{Институт экономики РАН, г. Москва, Россия}

Цель исследования. Современная макроэкономическая политика, опирающаяся на весьма сложные модели, казалось бы, достигла пика теоретического совершенства, однако, сила ее влияния и успешности оставляет желать лучшего. Многочисленные оченки, что экономическая политика не срабатывает, не приводит к результату - достижению иели, заставляют вернуться к истокам - принципу «цели-инструментьл", рассмотрев его расширенную трактовку и критику. Целью исследования выступает теоретический анализ модельно-инструментальных проблем разработки экономической политики с выявлением «эффекта превышения сложности», который в действительности детерминирует весь набор дискуссий в этой области, с помощью чего обосновывается необходимость применения принщипа иелей и инструментов.

Методологию исследования представляет собой теория экономической политики Я. Тинбергена.

Результатом исследования является обоснование ограничений в «критике Лукаса», а также в «эффективной классификации рынка» Р. Манделла, которая длительное время рассматривалась как расширенный вариант принщипа целей и инструментов. Представлена модель системной динамики, позволяющсая продемонстрировать наличие связей между ее параметрами и обнажающая невозможность учета всего набора механизма обратных связей. Вместе с тем полезность прямых формулировок, без учета всего набора связей, является важным инструментом для получения базисньх соотношений и выявления основных свойств системы, которые можно затем корректировать с учетом добавочных обстоятельств. Наличие у моделей обратного свойства к «эффекту превышения сложности» иногда обладает большей полезностью, нежели завышенная сложность, ограничивающая адекватность модели в большем масштабе, чем простота. Экономическая наука пытается бороться со сложностью посредством увеличивающейся специализации знания, но это должно означать дифференциацию инструментов в стремлении достичь взаимно детерминирующих иелей развития.

Ключевые слова: теория экономической политики Тинбергена; «критика Лукаса»; «эффективная рыночная классификация»; системные эффекты экономической политики; модели системной динамики; эффект превымения сложности.

\section{MACROECONOMIC POLICY: EFFECT OF EXCEEDING DIFFICULTY}

\section{(C) 2021 O. S. Sukharev}

\section{Institute of Economics, Russian Academy of Sciences, Moscow, Russia}

The aim of the study. Modern macroeconomic policy, based on very complex models, seems to have reached the peak of theoretical perfection, however, the power of its influence and success 
leaves much to be desired. There are numerous assessments that economic policy does not work, does not lead to a result - the achievement of the goal, they force us to return to the origins - the principle of "goals-tools», having considered its expanded interpretation and criticism.

The purpose of the study is a theoretical analysis of the model-instrumental problems of developing economic policy, with the identification of the "effect of exceeding complexity", which in fact determines the entire set of discussions in this area, with the help of which the necessity of applying the principle of goals and instruments is substantiated.

The research methodology is Tinbergen's theory of economic policy.

The result is a substantiation of the limitations in «criticism of Lucas», as well as in Mundell's "effective market classification», which has long been viewed as an extended version of the principle of goals and instruments. A model of system dynamics is presented, which makes it possible to demonstrate the presence of relationships between its parameters, revealing the impossibility of taking into account the entire set of feedback mechanisms. At the same time, the usefulness of direct formulations, without taking into account the entire set of relationships, is a useful tool for obtaining basic relationships and identifying the main properties of the system, which can then be adjusted taking into account additional circumstances. The fact that models have the opposite property to the "over-complexity effect» is sometimes more useful than overestimated complexity, which limits the adequacy of the model on a larger scale than simplicity. Economics tries to combat complexity through an increasing specialization of knowledge, but this must mean differentiating instruments in an effort to achieve mutually determinative development goals.

Key words: Tinbergen's theory of economic policy; «criticism of Lucas»; "effective market classification»; systemic effects of economic policy; models of system dynamics; effect of exceeding complexity.

Введение. Проводимая в России в течение первых двух десятилетий XXI века экономическая политика нередко подвергается критическим оценкам, особенно по аргументам недостаточной проработки ее стратегических ориентиров [1], недостаточного темпа роста [8], который она обеспечивает и который поддерживался, например, в советский период [2], отсутствия требуемого объема инноваций, высокого оттока капитала, обедняющего страну и дестабилизирующего финансово-валютный рынок [3-4], нехватки инвестиций и структурных дисбалансов [10].

Поиск причин неудовлетворительного развития всегда оборачивается выделением неких объективных обстоятельств функционирования экономики. Однако, когда ресурсный и человеческий потенциал у экономики имеется, «объективность» отрицательного исхода в развитии формируется в силу применяемых мер макроэкономической политики, точнее, необоснованной и неоправданно применяющейся политики. В таком случае имманентные ограничения моделей макроэкономики и выстраиваемых на их основе мероприятий, воздействующих на развитие, к сожалению, не принимаются во внимание. В итоге работает «эффект кобры», когда, влияя на экономику со стремлением придать ей новую динамику, наоборот, усиливают негативное проявление стагнации по причине применяющегося стереотипного набора мероприятий правительственной политики, ввергающих экономику именно в такое состояние ${ }^{1}$. Причиной становятся множественные обратные связи и системные эффекты реак-

1 Примером выступает «парадокс бережливости», когда стимулирование сбережений с целью использования их на рост увеличивает рецессию, понижая темп роста. Создание стимулов для сохранения занятости, но не за счёт повышения заработной платы, может привести к росту фрикционной безработицы, когда агенты ищут иные альтернативы труда, с более высокой заработной платой. П. Кругман так объясняет «парадокс гибкости», ратуя, что гибкие рынки труда, где бы легко понижалась заработная плата в условиях депрессии, являются обременяющим обстоятельством [13, с. 31-32]. Механизм влияния или передаточный механизм экономической политики объясняется так: снижение заработной платы понизит инфляцию, что приведёт к снижению процен- 
ции на сумму воздействий, предполагающие не только прямую связь параметров, по которым отслеживаются изменения, но и более сложные обратные связи.

Проблема измерения экономического развития [19] вместе с ограниченностью неоклассических макроэкономических моделей роста и рынков ${ }^{2}[9,14]$ закономерно приводит к видоизменению взглядов на депрессию, под которой, согласно Полу Кругману, следует понимать не просто глубокий спад, a функционирование, причем длительное, экономики ниже своих возможностей [13]. Данное состояние сопровождается тем, что стандартная макроэкономическая политика становится неспособной изменить ситуацию. Наоборот, балансирование бюджета и подавление инфляции могут закреплять депрессивное состояние, и поиск новых правил для выправления ситуации не обеспечит быстрого положительного результата. В связи с этим В. Смит утверждал ${ }^{3}$, что денежно-кредитная политика, а также фискальная политика не будут иметь силы влияния при отрицательной стоимости капитала [13], хотя фискальная политика может позитивно влиять на инновации и рост [16], а институциональные изменения определенным образом влияют на макроэкономические параметры функционирования [21], обнаруживая обрат- ную связь, которая не принимается во внимание в неоклассических моделях.

Суммируя, отметим, что изменение трактовок базовых понятий и влияния мер макроэкономической политики происходит под влиянием раскрытия сложности экономики, когда многие используемые модели превышают эту сложность или не дотягиваются до нее, что в обоих случаях составляет большой минус с точки зрения экономического анализа ситуации.

В связи с этим целью исследования становятся модели и процедуры формирования экономической политики (для обоснования передаточного механизма $\left.{ }^{4}\right)$, представленной набором инструментов, применяемых для достижения целей хозяйственного развития. Методологию исследования составляет теория экономической политики Яна Тинбергена с критикой Р. Лукаса $[11,15,17,19-20]$, которая заметно пошатнула подход с позиций применения больших эконометрических моделей, но отнюдь не отменила его полезности, особенно при отсутствии действенных альтернатив. Эффективная рыночная классификация Р. Манделла [5-6] лишь уточнила отдельные моменты стандартной теории экономической политики, привязав инструменты к целевым параметрам политики ${ }^{5}$, не снимая проблем, указанных Р. Лукасом. Особый

тных ставок и росту совокупного спроса. Критика неоклассического объяснения даётся в том, что при депрессии ставки процента и так низки - снижать их некуда, поэтому, по всей видимости, возрастёт безработица. Однако не приводится возражение, почему со снижением заработной платы должна понизиться инфляция, если заработная плата, скажем, составляет в доходах населения не выше 25-30\%, а также что побудит заработную плату к понижению в условиях несовершенных рынков труда. Когда объясняется тот или иной передаточный механизм влияния, значение имеет исходная точка - снижение заработной платы в приводимом объяснении. Нужен подробный анализ причин и правдоподобия такого снижения, да и величина снижения будет иметь значение. Если даётся объяснение, исходя из некоторых условий, то отсутствие условий будет означать практически автоматическое отсутствие и данного объяснения, либо понадобится добавочно объяснять, почему условий нет, а объяснение все равно имеет место быть, являясь правдоподобным.

2 В частности, рынков труда, рассматриваемых с точки зрения обеспечения равновесной нормы безработицы, с обоснованием единственности такой нормы для экономики в целом на уровне «гарантирующих допущений» [9]. В таком случае именно подобные допущения становятся проводниками не только решения макроэкономической задачи, но и предопределяют меры экономической политики. Проблемы безработицы, безусловно, важны, но они в определяющей степени являются производной проблем обеспечения занятости в экономике. Однако, меры политики, направленные на обеспечение занятости и элиминирование безработицы или негативных её эффектов все-таки связаны, хотя и отличаются по существу. Равновесные представления рынков, даже с учётом нестационарных проявлений, тем не менее, не позволяют учитывать всю гамму институциональных возможностей. Аккумулирующий эффект экономической политики обычно также не рассматривается в рамках поиска подобных рыночных решений, расположенных около или в точке равновесия.

3 По существу, речь идёт об особом институциональном состоянии макроэкономики - долговой экономике, когда обязательства превышают активы у большинства агентов, имеется кризис бухгалтерского баланса [13].

4 Под передаточным механизмом макроэкономической политики понимается последовательность (алгоритм) передачи воздействия инструмента на изменение целевого параметра политики.

5 Однако подобная жёсткость привязки снижает гибкость применения инструментария макроэкономической политики, когда один инструмент влияет на достижимость нескольких целей, причём действуя в положительном и отрицательном (тормозящем) направлении. 
акцент сделаем на системную реакцию, представляющую собой обратные связи различных изменяемых показателей. Это хорошо обнаруживается в подходе Дж. Форрестера и Д. Медоуза [7, 12] Представим аргументы, согласно которым и «критика Лукаса» содержит внутренние ограничения, не преодолевающие «эффекта превышения сложности» ${ }^{6}$, объективно присущего моделям макроэкономической политики. Низкая степень обоснованности мер политики, не учитывающая наличие обратных связей, реакций, формирует обратный к «эффекту превышения сложности» исход. И в том, и в другом случае результат применения политики один и тот же она не изменяет развитие в желательном направлении, но только по разным причинам, либо приводит к реакции, которая усугубляет проблемы («эффект кобры»), поэтому требуется выяснить, насколько имеющийся теоретический аппарат позволяет получать обоснованные воздействия на экономику.

1. Возврат к теории Тинбергена. Теория экономической политики Я. Тинбергена [20] предполагала получение системы эконометрических уравнений, связывающих цели политики и ее инструменты, причем оценивалась возможность решения системы дифференциальных уравнений, наличие связности инструментов. Уравнения (балансовые, технологические, институциональные и поведения) отражали связи между переменными экономики посредством коэффициентов влияния. Решение позволяло получить оценку того, как именно развивается экономика под воздействием некоего набора предпринимаемых решений. Данный подход применялся для моделирования политики роста [11], планирования развития [19]. Вместе с тем связность целей, а также взаимная детерминация инструментов политики никак не преодолевались в рамках этого теоретического подхода. Конфликтовали не только цели, но и инструменты, а планирование требует постоянного изменения информации и коррекции самих планов, включая и инструменты воздействия.
Таким образом, при разработке несложных моделей, пригодных в управлении, оказывалось, что «структурная статика» таких моделей никак не преодолевается. Инструменты экономической политики, будь то инвестиции, распределяемые по направлениям деятельности, госрасходы, налоги, субсидии, процентные ставки или пошлины, несмотря на институциональную детерминацию, зависимы во влиянии от многих иных обстоятельств, изменчивость которых может не отражаться в зафиксированном уравнении или системе уравнений. Цели развития также многочисленны, например, это и рост валового продукта, доходов населения, численности населения, торможение динамики цен, снижения издержек и др. Как видим, задачи максимизации совмещены с поиском минимума, причем достижение одной цели может нивелировать достижение другой, особенно при обратном соотношении целей. Улучшение теории Тинбергена за счет использования функций благосостояния и социальных потерь, учета издержек применения инструментов, а также элиминирования неопределенности применения инструментов, тем не менее, не снимает важности проблемы выбора инструмента экономической политики в тех или иных условиях развития. Особо сложной выглядит задача применения целого набора инструментов. Дополнительным к принципу Тинбергена [20] выступает принцип эффективной классификации рынка Р. Манделла [5-6], согласно которому каждый инструмент приспособлен для своей цели наилучшим образом и должен применяться для соответствующей цели [5].

Однако здесь возникает проблема обоснования предназначения инструмента политики исходя из теоретической модели или из сложившихся и исследуемых конкретных условий. Дело в том, что Р. Манделл рассматривал внутреннее (совокупный спрос равен совокупному предложению при полной занятости) и внешнее (торговый баланс покрывает экспорт капитала при данном паритете обменного курса) равновесие при некоторых допущениях (например, запасы

6 Когда создаваемые модели, с помощью которых обосновываются меры воздействия на экономику, настолько сложны, что реальность для понимания более простая, нежели сама модель, возникает эффект превышения сложности. 
считались полностью исчерпанными). Тем самым точка равновесия признавалась наиболее приемлемой, куда и должны привести экономику применяемые инструменты денежно-кредитная и бюджетно-налоговая политика.

Следовательно, текущая ситуация оценивалась по тому, насколько экономика удалена от равновесия, а цель политики вытекала автоматически - привести ситуацию к равновесию за счет указанных инструментов. При этом не учитывалась даже мера исходной неравновесной ситуации, которая не может не сказаться на силе и масштабе применяемых инструментов и присутствует на практике, а не на уровне модели. При таком представлении проблемы неясным остается и то, насколько велико принуждение инструмента экономической политики по движению к равновесию по его обеспечению и удержанию ${ }^{7}$. Исходя из основных допущений [5], было показано, что монетарная политика пригодна для обеспечения равновесия платежного баланса (внешнее равновесие), бюджетноналоговая - для обеспечения внутреннего равновесия. Однако, при их одновременном применении возможны ситуации, когда оба вида политики будут мешать друг другу либо тормозить с учетом реакции системы движение ее к равновесным, пусть и модельным, ситуациям.

Таким образом, указанная дихотомия с привязкой инструмента к цели на практике мало что давала для выстраивания экономической политики длительно неравновесной развивающейся экономики. К тому же не учитывались обратные связи и влияния различных параметров. Фиксировались стереотипы проводимой ранее политики, а именно: когда при активном сальдо платежного баланса снижали госрасходы, увеличивали налоги и проводили политику дешевых денег (снижали процент), а при дефиците платежного баланса, наоборот, снижали налоги, удорожали деньги (повышали процент), увеличивали расходы. Такие действия не предполагали рассмотрения, когда повышение налогов может обострить дефицит бюджета, понизить расходы, или снижение налогов привести к такому же исходу. Разведение влияния инс- трументов, в частности, процента и налогов на внешнее и внутренне равновесие может не подтверждаться динамикой параметров в конкретных экономических условиях. Это относится к экономике, где изменение процентной ставки будет сильно влиять на инвестиции и сбережения, причем на первую компоненту сильнее, чем на вторую. Со снижением процентной ставки за счет роста инвестиций возможно увеличение бюджетного профицита (при высокой эффективности инвестирования), рост же процента обеспечит нивелирование профицита бюджета, переводя его к некоторому дефициту (зона OEA на рисунке 1). Возможна ситуация, что релевантной связи процентной ставки и бюджетного дефицита/профицита не будет. Более того, на относительно коротком интервале времени связь параметров может быть одна, на ином отрезке времени - совершенно иная. Учитывая, что экономическая политика ориентируется на текущее применение инструментов, конечно, определяющим выступает непродолжительный отрезок времени.

Вид кривой $\mathrm{OE}$ может оказаться любым - для каждой страны своим собственным, включая и отсутствие какой-либо детерминации между изменением налогов и сборов и процентной ставкой. Рост процентной ставки может сопровождаться снижением дефицита бюджета и переходом на профицитный бюджет в точке i1 (штриховая линия на рисунке 1). Такие связи и виды динамики требуют объяснения конкретными условиями развития экономики.

Критическое отношение к теории Я. Тинбергена было изначально оформлено Р. Лукасом [17] и сводилось к тому, что статистика прошлого периода не справляется с изменяющимися связями целей и инструментов. В уравнениях эконометрических моделей коэффициенты связи должны изменяться, не оставаясь стабильными так, как это отвечает реальности. Кроме того, можно отметить и лаги в сборе и использовании той или иной статистики. Конечно, искажения сопровождают процесс моделирования на каждой его стадии, включая подготовку данных, отбор переменных, установление связей, поиск решения и его интерпретацию. Инструмен-

7 В связи с этим остаётся неясно, насколько необходимо стремиться к такому результату. 
ты политики применяются одновременно, но сами изменчивы по силе и охвату применения, а также синхронности воздействия на экономику. На рассматриваемом интервале времени они могут многократно изменяться в соответствии с реакцией экономики на их применение и изменение целевых показателей. По идее, в таком случае должна измениться и модель, которая уже не будет отражать связи целей и инструментов. Хотя если модель делается с некоторой степенью неточности (иначе она бы не была моделью), то добавочные искажения, наслаиваемые следующими периодами времени и изменением самой экономики и ее реакции, могут оказаться вполне удовлетворительными, если промежуток времени не очень продолжительный. Однако предложение использовать ожидаемые значения переменных моделей вместо прошлых данных с привязкой к адаптации не решает названной проблемы, так как изменение политики должно изменить и ожидания [17]. Строгость оценки рациональных ожиданий, характеризующих непредвиденное изменение параметров модели, весьма не- высока, предполагает оценку свершившегося ожидания в текущем периоде, но ожидание осуществлено периодом ранее (в прошлом). Оливер Бланшар [15] опроверг позиции Лукаса ${ }^{8}$ по поводу влияния изменений в инструментах политики на связующие коэффициенты эконометрической модели. Вместе с тем имеющая подтверждения в отдельных примерах теория Лукаса не дает ответа на вопрос о формировании ожиданий, как и о связи их с коэффициентами модели. Более того, ожидания двух рядом соседствующих периодов могут отличаться довольно существенно, как и влиять или не влиять на параметры модели. Важно и то, каким способом осуществлять ожидание по разным переменным. Ожидание инфляции - это один параметр, а ожидание инвестиций или изменения процентных ставок - совсем иной.

Таким образом, на уровне создания моделей заложена имманентная сложность, связанная с математической записью модели и трактовкой параметров, в нее входящих, статистическим измерением этих параметров. Видимо, она возникает по той причине,

\section{Бюджетный профицит (налоги)}

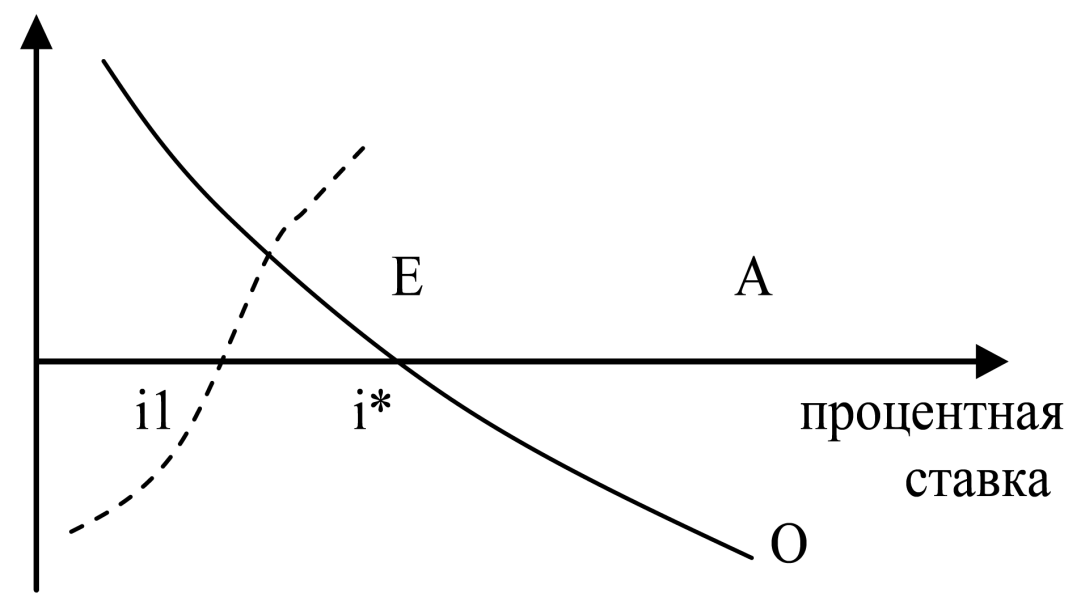

\section{Бюджетный дефицит}

Рис. 1. Бюджетный профицит/дефицит и процентная ставка

8 Тем самым «критика Лукаса» серьёзно не пошатнула теории Тинбергена, сохранив общий подход в области эконометрического моделирования экономической политики, лишь добавив туда элементы учёта ожиданий (адаптивных и рациональных). Однако весьма трудно доказать, что главенствует рациональное ожидание и что оно правильно учитывается в расчёте или пересчёте изменяющихся коэффициентов. Точного аппарата, который бы позволял найти, что коэффициенты, например, не изменились, а также реакция на инструменты, но ожидания стали иными, насколько известно, не существует. 
что стереотипные математические методы моделирования не позволяют учесть всю гамму обратных связей переменных, целей и инструментов, причем изменяющуюся во времени. Попытки учета обратных связей предпринимались в моделях управления и моделях системной динамики [7, 12]. Рассмотрим их подробнее, раскрыв проблему «эффекта превышения сложности».

\section{2. Эффект превышения сложности:} структура целей и инструментов. «Эффект превышения сложности» иллюстрируется структурными изменениями и реакциями экономики на их совместное с макроэкономической политикой влияние. Если формируемая модель использует допущение, которого нет в реальности, она сразу порождает необходимость дальнейшей интерпретации именно этого допущения с точки зрения полученного результата и применения модели, ведь этот результат в данном случае получен для экономики с введенным допущением. Иначе невозможно объяснить, зачем оно вводилось (исключаем ошибочный ввод допущения или ограничения ${ }^{9}$ ). Превышение сложности связано еще и с высокой неопределенностью экономической ситуации, которая находит отражение в проектируемых моделях реальности, когда существующая неопределенность умножается на неопределенность модели. Наличие структуры целей и совокупности инструментов обеспечивает эту неопределенность.

Макроэкономическая политика, ориентируясь на агрегированные показатели, совершенно не ориентируется на то, какая экономическая структура будет способствовать развитию. Более того, не принимается во внимание и то обстоятельство, что различное распределение ресурсов в экономике может дать несовпадающее соотношение дохода и риска ведения деятельности с высокой неопределенностью проблемы выбора структуры распределения.

Влиять на риск и доходность можно, например, институциональными коррекциями (демпфирование риска), либо предоставляя дополнительный ресурс в рамках программ развития и национальных проектов. Также будет способствовать решению такой задачи создание стимулов для дополнительного инвестирования в наиболее рискованном и менее доходном направлении деятельности, которое очень необходимо с точки зрения хозяйственного развития. Стереотипные рыночные подходы фактически лишили бы его ресурса на развитие, потому как рискованное и низкодоходное дело не привлекает ресурса и инвестиций.

Выбор экономической структуры показан на рисунке 2, где кривые I и II соответствуют различным структурам распределения ресурса по объектам экономики, дающим некую величину дохода и риска. Однако возникает ситуация в так называемых характерных точках 1-2-3, где разные структуры распределения ресурса дают одно и то же сочетание дохода и риска, даже вблизи этих точек. Это пример превышения сложности - модель не дает решения (будь то максимизация дохода или минимизация риска $\left.{ }^{10}\right)$, так как выбор крайне неопределенный. Нужны либо добавочные качественные критерии, либо некие эксперты, совокупность мнений которых удастся обработать. В качестве критерия выбора может использоваться темп роста экономики в характерной точке, например, выбирается то распределение, для которого темп роста в характерной точке будет выше.

В линиях связи (штриховые III и IV на рисунке 2) выбор в районе точек C, D так же неопределенный, как и в точках 1-2-3, однако выбор между характерными точками C и D вполне определен в пользу большего дохода и меньшего риска - точка D. Для характерных точек 1-2-3 данный вопрос также неразрешим, определяется соотношением величины дохода и риска. Конечно, можно сказать, что если структурный выбор так труден и неочевиден, зачем его осуществлять? Однако он сильно влияет на развитие, зависим от проводимой макроэкономической политики, детерминирующей перемещение ресурсов в экономике, в частности, инвестиций. Представим, что структура инструментов,

9 При ошибочном допущении модель может не работать, а может сработать, так как допущение или ограничение не является релевантным.

10 Применение каждой из оптимизационных моделей даст решение, которое будет по постановке оптимальным, но по содержанию не составит полезность для развития экономики. 
изменяясь, дает различную величину дохода и риска в экономике. Именно эти инструменты и означают распределение ресурса по экономическим объектам.

Однако не так прост выбор структуры распределения и вне характерных точек, хотя в них его трудность очевидна. Например, нужно выбрать структуру распределения, дающую доход по линии А, где имеется невысокая доходность. Если рассматривать выбор по линии А, то выбирается при том же доходе структура, дающая меньше риск (при прочих равных). Если рассматриваем выбор по линии В, применяем ту же логику. Однако, сопоставляя выбор по двум линиям А и В, видим, что для линии В доход выше, но и риск выше, чем при выборе структуры по линии А. Следовательно, выбор структуры, видимо, будет определяться относительным превосходством дохода и риска в двух случаях, а также набором иных факторов (качественного характера).

Представим теперь, что структура распределения ресурса полностью определяется макроэкономической политикой - ее инструментами. Тогда представленная задача выбора - это выбор структуры инструментов, позволяющих достигать определенную структуру целей. Тем самым можно привязать структуру инструментов к распределению ресурсов и показать, какие цели и как удается достигать. Это также будет свое- образная модель экономической политики, но включающая фактическое состояние элементов экономики под влиянием поступающих к ним и перерабатываемых ресурсов.

Иными словами, «эффект превышения сложности» имеет основу не только в моделировании - представлении экономической динамики, связи инструментов политики и целей, но связан с имманентными особенностями функционирующих социальных систем.

Во-первых, макроэкономическая политика фиксирует проявления функционирующей экономики и обеспечивает воздействие на них, порождая тем самым ответную реакцию. Она и генерирует модель поведения экономики в рамках проводимой политики, заставляя ее изменяться. Однако совсем не факт, что применяемый набор инструментов улучшает, а не ухудшает функционирование экономики, либо является не самым лучшим вариантом (как это следует из рисунка 2).

Во-вторых, оперативные задачи макроэкономической политики, в частности, преодоление кризиса, вызванного форс-мажорной ситуацией распространения вируса, может затруднить реализацию долгосрочных целей, либо отодвинуть их во времени. Кроме того, реактивные свойства экономики на коротких и длинных отрезках абсолютно отличаются по природе. Решение текущих проблем может создавать новые

\section{Риск}

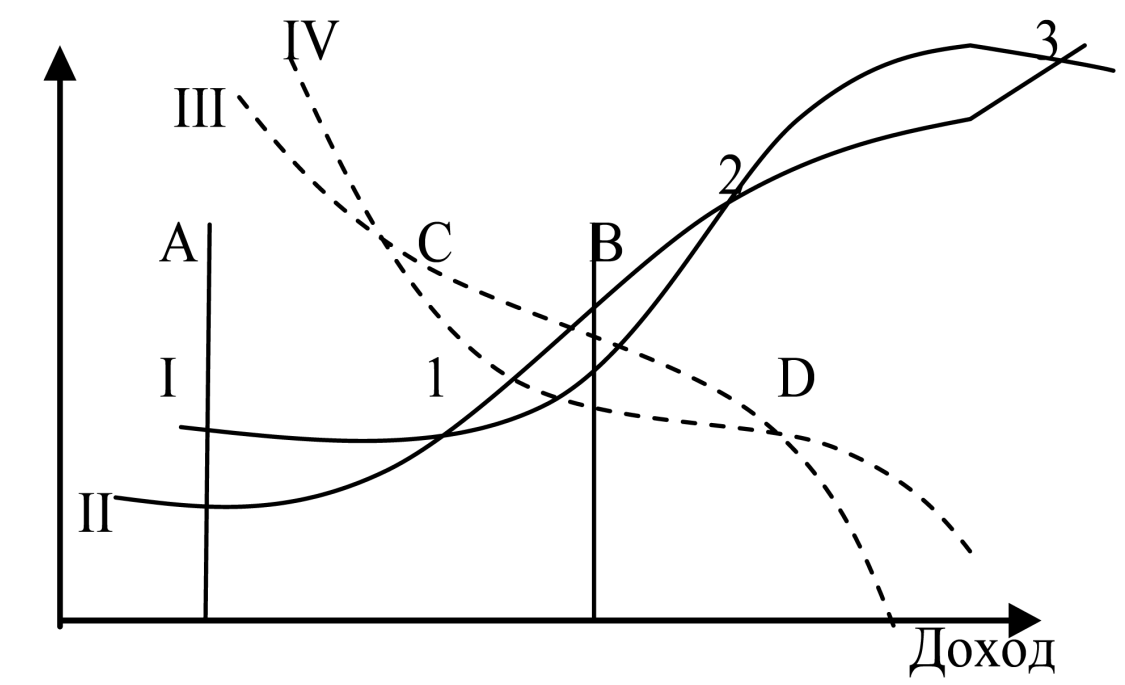

Характерные точки: $1-2-3$

Рис. 2. Выбор структуры инструментов - характерные точки 
в силу противоречивости целей и применяемых инструментов.

В-третьих, мероприятия макроэкономической политики, применяемые инструменты могут оказаться с низкой чувствительностью по влиянию на релевантные параметры экономики. Это приведет к низкой ресурсной отдаче и к фиксации ситуации без ее существенного изменения и достижения целей развития [12].

Названные три условия, так или иначе фигурирующих при создании моделей системной динамики, наглядно показывают источник «эффекта превышения сложности», вытекающий из общей неопределенности применения инструмента политики и изменения цели при наличии перемешивающего влияния. Скажем, обосновывается задача увеличения монетизации экономики в силу недостаточной величины денежной массы в объеме ВВП. Однако, предположим, в экономике по факту уже она происходила, только в диапазоне, скажем, от 14 до 58\% ВВП ${ }^{11}$. В связи с этим дальнейшая монетизация может либо сгладить эффект предыдущего ее действия на экономику, либо, наоборот, усилить эффект роста, но сопроводить его ускорением инфляции. Тогда подавление инфляции будет означать и торможение роста одновременно. Это будет верно при условии наличия связи между ВВП и конкретным уровнем его монетизации, а также соответствующим состоянием экономической структуры и финансовых институтов, принимающих эту увеличивающуюся денежную массу от $60 \%$ и выше. Таким образом, характер связи и воздействия инструмента на экономику наверняка будет зависеть от размера и силы применения инструмента, а также от состояния структур, воспринимающих воздействие этого инструмента, если они от него не могут не зависеть, как в случае с денежной массой.

Создание моделей предполагает помимо ввода неких допущений и ограничений, которые затем весьма нелегко интерпретировать, рассмотрение связей между элементами экономики. Рассматривая подобные связи, нужно понимать, как важны обратные реакции, которые часто не учитываются на математическом уровне, обеспечивая обратную позицию к «эффекту превышения сложности». Покажем это обстоятельство в рамках системной модели динамики (рисунок 3 ).

На рисунке 3 показано, что люди, генерируя знания, создают технологии, производство, которые исчерпывают природные ресурсы (невозобновляемые) для создания про-

\section{Расход}

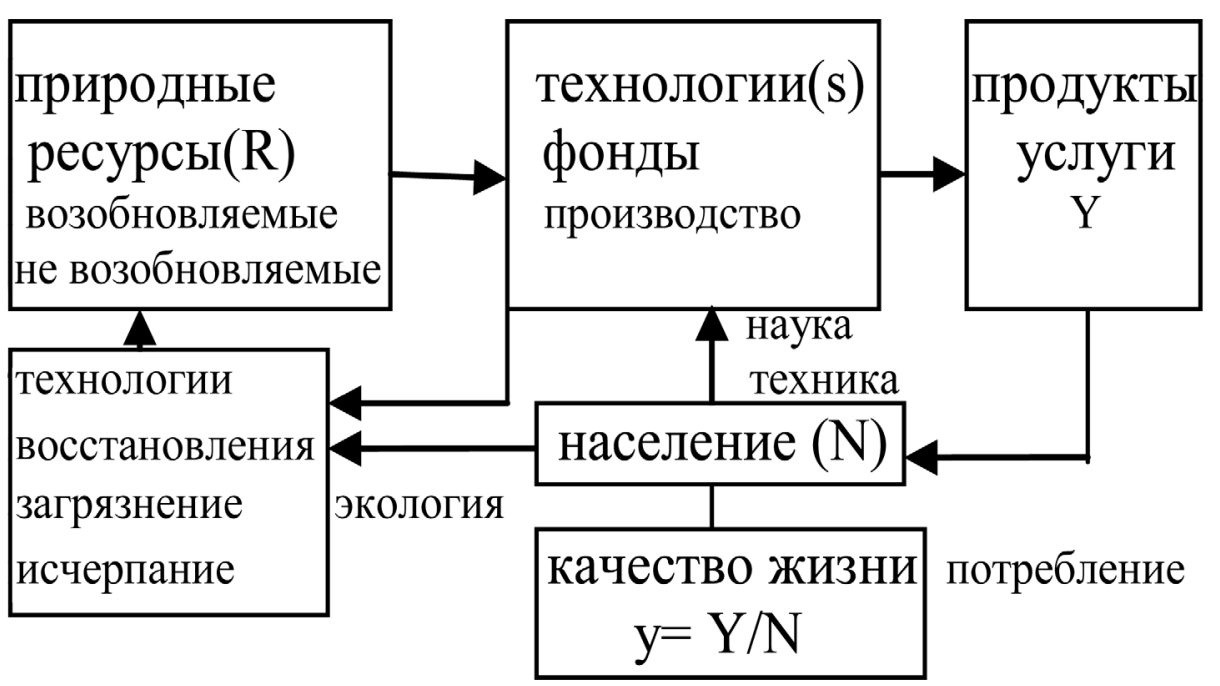

Рис. 3. Системная динамика экономики

11 Соразмерность подобного увеличения во времени, видимо, будет определять характер воздействия монетизации на экономический рост, но сам уровень монетизации и диапазон, в котором происходит его увеличение, безусловно, становятся самостоятельным инструментом влияния. 
дуктов и услуг, формирующих потребление и качество жизни. При этом возникают колоссальные экологические издержки, разрушающие ресурсную базу. Однако создаются и технологии, на которые тратится часть ресурсов, способные создавать новые ресурсы или уменьшать потери существующих ресурсов, либо восстанавливать отдельные виды ресурса (заменять). Вместе с тем экологические дисбалансы сказываются на увеличении численности населения $\mathrm{N}$ и на качестве жизни. На рисунке эта связь не показана, но она присутствует. Другое дело, насколько сила этого влияния велика в различные периоды развития цивилизации, при неуклонно возрастающей численности населения с некоей величины эти ограничения становятся весьма критическими для обеспечения парадигмы роста и удовлетворения потребностей в равном объеме все возрастающего населения. Более того, $\mathrm{N}=\mathrm{N}(\mathrm{Y})$, численность населения наверняка может быть функцией создаваемого продукта и качества жизни, причем зависимость от продукта на каком-то этапе может оказаться пропорциональной, а вот от качества жизни - обратно пропорциональной (темп роста численности затормозится при высоком качестве жизни). Тем самым по ходу развития меняются связи параметров и воздействие одной величины на другую. Консервация целей экономической политики при этом может привести к весьма неадекватным результатам с точки зрения управления развитием экономики.

Однако представленная схематично модель вполне отражает то, что происходит. Имеются ресурсы, они комбинируются, используются для производства благ, обеспеченность которыми становится основной для увеличения численности населения, что в свою очередь актуализирует задачу совершенствования технологий производства за счет науки и образования населения с тем, чтобы решать задачи удовлетворения увеличивающихся с ростом числа людей потребностей. Парадигма роста занимает в этой схеме центральное место, формируя экономическую философию жизни. Данная схема работает до тех пор, пока объем ресурсов не станет таким, что условия жизни значительного числа людей ухудшатся, а наука и технологии уже не смогут разви- ваться таким темпом, чтобы дать решения по компенсации утраченных ресурсов. Это будет подлинная трагедия общин, связанная со стремлением обеспечить рост и богатство в условиях больших, но все же ограниченных ресурсов. Если в виде цели положить рост эффективности $\mathrm{w}=\mathrm{Y} / \mathrm{R}$, то есть создаваемого продукта с единицы ресурса, с условием, например, возобновления ресурса, либо контролируемого исчерпания $\mathrm{dR}=\mathrm{b}$, то несложно получить условие роста эффективности. При такой постановке задачи эффективность растет при $\mathrm{dY} / \mathrm{dR}>\mathrm{w}$, или $\mathrm{dY} / \mathrm{b}>\mathrm{w}$, когда изменение продукта при фиксированном изменении ресурса на единицу этого изменения превышает текущую эффективность.

Записав трансформацию ресурса в продукт в виде $\mathrm{Y}=\mathrm{Rs}$, где $\mathrm{s}$ - научно-технологическая функция, учтя у $=\mathrm{Y} / \mathrm{N}=\mathrm{Rs} / \mathrm{N}$, получим условие роста продукта $\left(\mathrm{g}_{\mathrm{y}}>0\right)$ на одного человека: $\mathrm{g}_{\mathrm{N}}<\mathrm{g}_{\mathrm{R}}+\mathrm{g}_{\mathrm{s}}$, где $\mathrm{g}_{\mathrm{N}}, \mathrm{g}_{\mathrm{R}}$, $\mathrm{g}_{\mathrm{s}}$ - соответственно темп роста населения, расхода ресурсов и научно-технического прогресса. Таким образом, для роста душевого продукта темп роста численности населения должен быть меньше суммы темпов роста исчерпания ресурсов и научно-технического прогресса. Однако это соотношение как раз не учитывает обратные связи, о которых говорилось выше. Следовательно, без учета не просто обратных связей, но и их самого изменения получить точные правдоподобные оценки и соотношения весьма проблематично, хотя подобные весьма ориентировочные, но дающие представление об изменении общих агрегатов и структуры в экономике модели-схемы, включая и сложные эконометрические системы уравнений в рамках подхода Тинбергена являются весьма полезными ориентирами для принятия различных решений. Из представленной модели также следует, что, если ресурсы исчерпываются $\mathrm{dR} / \mathrm{dt}<0$, то темп научнотехнических изменений и технологической модернизации должен обгонять темп роста продукта. Конечно, такое соотношение будет справедливым при условии, что на саму технологическую модернизацию не понадобится значительный и нарастающий объем peсурса. Темп технического прогресса для роста душевого продукта должен обгонять 
взвешенную разницу ${ }^{12}$ скоростей пополнения ресурса (при открытии новых ресурсов) и его исчерпания.

Как видим, если целью развития выступает душевой продукт, то соотношения, которые бы могли обеспечить его увеличение, задают возможности структуры инструментов макроэкономической политики. Причем темп увеличения душевого продукта зависит и от того, как изменяется население, то есть определяется рождаемостью и смертностью. Известный закон изменения численности населения $\mathrm{dN}=(\mathrm{b}-\mathrm{d}) \mathrm{N}$ с решением $\mathrm{N}=\mathrm{N}(0)$ $\exp (b-d) t$, где b, d - рождаемость и смертность [12], будучи подставлен в условие роста душевого продукта $\mathrm{g}_{\mathrm{N}}<\mathrm{g}_{\mathrm{R}}+\mathrm{g}_{\mathrm{s}}$, даст следующее условие: $\mathrm{b}<\mathrm{d}+\mathrm{g}_{\mathrm{R}}+\mathrm{g}_{\mathrm{s}}$ или $\mathrm{g}_{\mathrm{s}}>\mathrm{e}-\mathrm{g}_{\mathrm{R}}-$ темп научно технического прогресса должен превышать разницу между естественным приростом населения (е) и темпом расхода ресурсов.

Действительно, выглядит правдоподобно для роста душевого продукта превышение темпа научно-технического прогресса темпа расхода ресурсов с противоположным знаком (это установлено, исходя из $\mathrm{Y}=\mathrm{Rs}, \mathrm{g}_{\mathrm{Y}}>0$ ).

Если ввести инвестиционную функцию продукта, скажем, $\mathrm{Y}=\mathrm{AI}^{\mathrm{a}}$, то $\mathrm{g}_{\mathrm{Y}}>\mathrm{g}_{\mathrm{N},} \mathrm{g}_{\mathrm{Y}}=\mathrm{ag}_{\mathrm{I}}$, откуда $\mathrm{g}_{\mathrm{N}}<\mathrm{ag}_{\mathrm{I}}$. Темп роста населения должен быть меньше взвешенного темпа роста инвестиций. При росте инвестиций показатель степени «а» больше отношения темпов роста населения и инвестиций, при инвестиционном спаде, соответственно, меньше, для обеспечения обнаружения роста душевого продукта. Как видим, простейшие модели также имеют свое полезное назначение структурирование проблемы и поиска путей ее разрешения.

Пытаясь установить правдоподобие связей между величинами, целями и инструментами, исследователи, как правило, увеличивают сложность, не снимая ее. В итоге улетучиваются такие показатели эффективности научной работы, как время получения резуль- тата и затраты на его получение ${ }^{13}$, особенно относительно оценки тех возможностей, которые дал сам результат. В заключение рассмотрим, как наука (не только экономическая) в силу канонов своей самоорганизации реагирует на высокую сложность, возрастающую с ростом полученного ею знания.

\section{3. Специализация и дифференцирова-} ние преодолевают сложность. С ростом объема знаний увеличивается сложность их использования и обучения, на что закономерно увеличивается специализация знания (рисунок 4, вверху). Появляются отдельные дисциплины и направления науки, причем этот процесс идет по разным траекториям $(1,2$ на рисунке 4, вверху). Линия 1 отвечает сначала быстрому течению процесса, затем замедлению, линия 2 - сначала процесс идет медленно, затем резко ускоряется вплоть до своего предела в виде некоего потолка знаний. Траектория DFG возникает при появлении абсолютно нового знания, когда уже, казалось бы, основные позиции известны. Если исчерпано знание $\mathrm{K}^{*}$, то специализация может возрасти по линии $\mathrm{BC}$ за счет внутренней отстройки знаний, упорядочения, методико-таксономической работы самих исследователей. Возможно развитие по траектории ВА, когда знание увеличивается, но специализация его не растет, так как для увеличения различных направлений требуется больший объем знаний.

Итак, можно говорить о том, что рост знаний в рамках какой-то науки будет в среднем способствовать росту ее специализации $\mathrm{H}=\mathrm{f}(\mathrm{K})$ (рисунок 4 внизу). В области теории экономической политики с насыщением знаний о влиянии инструментов на экономику можно предположить, что понадобится дифференциация инструментов в зависимости от уровня специализации знаний в этой области, то есть $\mathrm{DF}=\mathrm{V}(\mathrm{dH} / \mathrm{dt})$, это и будет закономерным результатом преодоления сложности. Иными словами, чем больше исследователь знает о влиянии отдельного

12 Взвешивающий коэффициент представляет собой обратную величину текущему объёму используемого pecypca.

13 Российской традицией стало обстоятельство, согласно которому можно получать деньги на разработку некой модели, чуть совершенствуя её на протяжении десяти лет, ровным счётом ничего не привнося в теоретическом плане, а также в области собственного моделирования и тем более решения практических вопросов макроуправления. Конечно, поддержка этой работы может дать и некий прорыв, но неопределённость этого прорыва и эффект превышения сложности обычно обесценивают подобные усилия и ожидания в экономической науке. 
инструмента, тем сложнее учесть это влияние и получить полноценную картину воздействия набора применяющихся обычно одновременно инструментов экономической политики, особенно если принять во внимание значение состояния экономических институтов и структурные аспекты влияния и реакции экономики. Тем самым влияние экономической политики будет становиться все более дифференцированным, что не может не отразиться на теоретическом и практическом подходе, применяющихся для ее обоснования и реализации.
В последнее время на правительственном уровне помимо стандартных мер экономической политики (бюджетно-налоговой и денежно-кредитной) все активнее применяются различного рода институциональные коррекции. Сразу отметим, что они с большим трудом подлежат моделированию и какому-то предсказанию их эффективности, а указание на чей-то опыт успешного применения института не является индульгенцией и точным обоснованием необходимости ввода этого института в других условиях, на ином историческом интервале и в другой

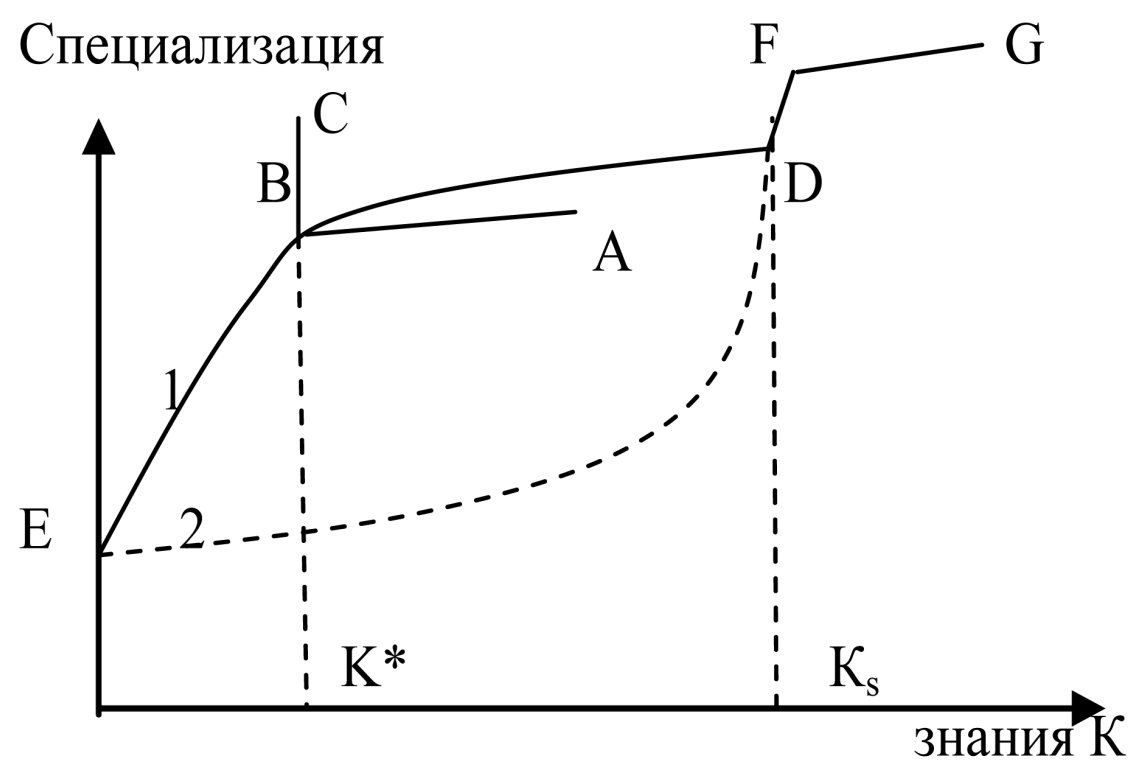

\section{Дифференциация инструментов}

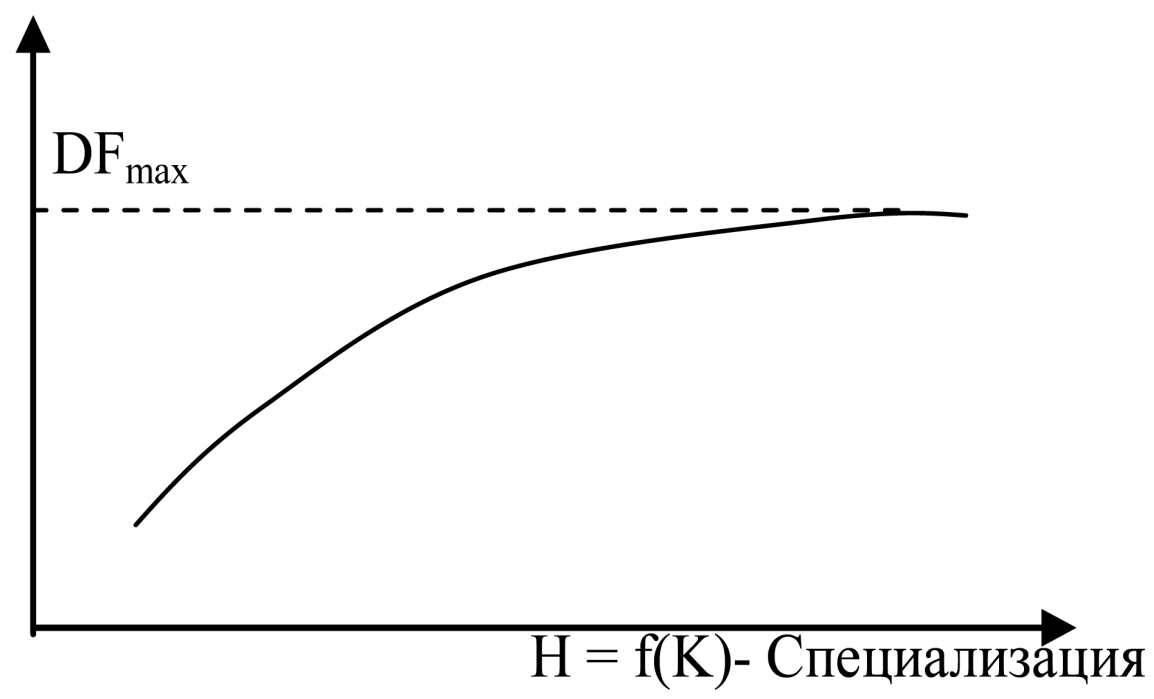

Рис. 4. Специализация знания и дифференциация инструментов политики 
стране. Управляемое изменение институтов обычно проводится по двум основным направлениям: меняются (корректируются) внутренние институты, либо вводятся новые правила, каким-то образом взаимодействующие с прежними институтами, а также вводятся институты, которых не было в данной стране, но использовавшиеся в других странах, то есть происходит импорт институтов. При импорте институтов осуществляется трансформация действующих внутри страны институциональных структур. Влияние каждого института особенное, так как каждый из них имеет свое содержание, назначение, приносит определенную полезность. Другое дело, можно вести речь о предельной полезности следования одному и тому же институту, то есть исполнению этого правила. С ростом числа исполнений и течением времени предельная полезность следования, то есть каждого последующего исполнения правила, может, например, убывать. Но она может и возрастать, в частности, в силу осуществленной коррекции и некоего усиления работы этого правила. Поскольку институты крайне неоднородны, говорить об их собственной предельной полезности в привязке к числу институтов не приходится ${ }^{14}$. Полезность института в каждом случае своя собственная. Однако вполне возможно вести речь о полезности акта заимствования в сравнении с актом создания собственного правила внутри страны в зависимости от числа заимствуемых институтов и частоты изменения внутренних институтов (см. рисунок 5).

Сопоставление затрат на импорт институтов и внутренние изменения могут быть критерием для принятия решения, но такой критерий действует весьма ограниченно. Значение имеют не только затраты, но и приобретаемый - ожидаемый доход от изменения институтов за счет импорта или внутренних коррекций. Получаемый доход зависит не только от затрат на изменение институтов, но и от скорости самих изменений, частоты смены правил. Таким образом, принятие решения может детерминироваться своеобразным институциональным мультипликатором. Он показывает, как изменяется ожидаемый доход от институциональных коррекций - внутренних и за счет импорта институтов (раздельно или вместе), если затраты возрастают на единицу, либо интенсивность институциональных изменений увеличивается на единицу.

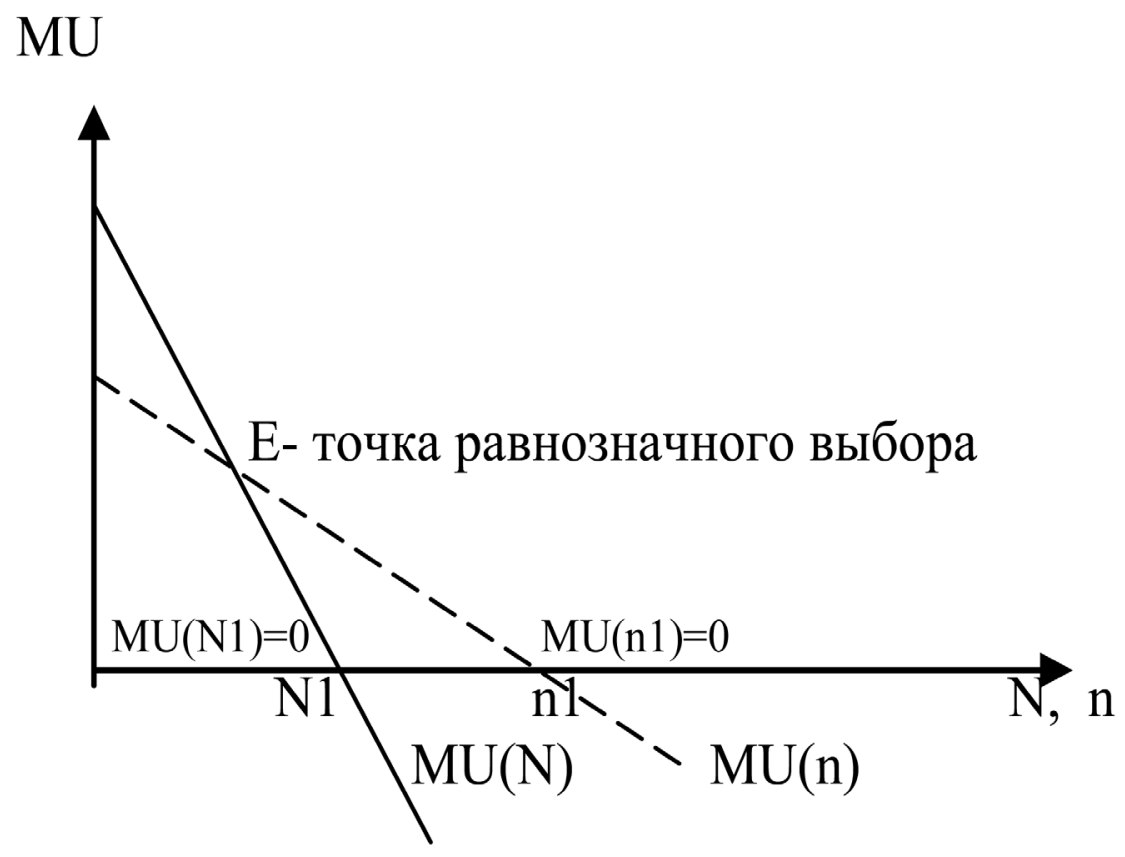

Рис. 5. Предельная полезность актов заимствования и внутренних изменений институтов

14 Институты могут рассматриваться как общественное благо, но всё-таки не являются яблоками или пирожками, на примере которых в стандартных микроэкономических работах анализируется предельная полезность блага. Хотя некий схемно-модельный вариант представления, видимо, возможен. 
Рисунок 5 отражает графическую модель, показывающую изменение предельной полезности от растущих актов заимствования институтов $(\mathrm{N})$ и от частоты (n) внутренних институциональных коррекций. Причем показан случай, когда предельная полезность снижается с ростом числа актов импорта институтов и увеличением частоты внутренних изменений - коррекций. В точке равновесия частота внутренних изменений и число актов импорта институтов такие, что обеспечили одинаковую предельную полезность. Если критерием выбора является предельная полезность, то в этой точке выбор равнозначен - можно осуществлять либо импорт, либо внутренние изменения. Левее точки Е по этому критерию выгоднее импортировать институты, правее точки Е - осуществлять внутренние коррекции.

Причем в этом случае скорость институциональных реформ должна превысить ту скорость, которая отвечает равновесной точке. Если критерий принятия решения величина затрат к ожидаемому доходу, тогда по каждому импортируемому институту и корректируемому внутри страны должны сопоставляться эти коэффициенты. Однако получить ожидаемый доход, не пройдя путь функционирования данного института, невозможно. Ни одна модель не даст такого предположительного результата. В связи с этим и имеются налицо фундаментальные ограничения по учету институциональных факторов в описании как роста - на любых моделях, так и в принятии решения по выбору траектории институциональной трансформации.

По этой причине, принимая те или иные меры в рамках экономической политики, ориентируются на общие возможности позитивного влияния на развитие экономики. Задача же взаимного усиления или ослабления различных инструментов в условиях многоцелевой системы на интервале, превышающим краткосрочный горизонт, по существу, не решалась и остается нерешенной в современной теории экономической политики. Например, рост инвестиций в основной капитал может сопровождаться снижением рентабельности продаж, а соотношение рентабельности и риска быть таким, что большему доходу не будет соответствовать больший риск, а меньшему доходу - меньший риск его получения. Причем ситуация возникает по ходу развития экономики и требует иного — структурного - подхода [18] в области теории экономической политики вместо классического варианта целей и инструментов.

Заключение. Подытоживая проведенный теоретический анализ, приходим к следующим позициям.

Во-первых, сегодня недостаточно привязки инструментов экономической политики к целям и ответственности каждого инструмента, который лучше всего обслуживает какую-то цель. Также в силу изменчивости ожиданий и сложной их природы в силу присутствия и адаптивного, и рационального начала коэффициенты эконометрических моделей не могут иметь гарантии верификации при изменении экономической политики и реакции экономики на нее.

Во-вторых, сложность механизмов обратной связи делает записи самих уравнений весьма условными, как и полученные решения, а проблема выгодного управления сводится к минимизации функционала Понтрягина, не снимая, а актуализируя задачу быстродействия, то есть действенного применения инструментов за как можно меньшее время. В этом случае она перестает быть частным случаем. Базовые уравнения, описывающие экономику, должны включать функцию управления в виде $\mathrm{dY} / \mathrm{dt}=\mathrm{f}(\mathrm{Y}, \mathrm{z})$, где $\mathrm{Y}$ - продукт, Z - функция управления (инструменты макроэкономической политики). В итоге тот, кто быстрее ликвидирует последствия «ковидного кризиса», будет иметь преимущества в развитии и в дальнейшей конкурентной борьбе.

В связи с этим подход к макроэкономической политике должен становиться все более дифференцированным, включать попытки взвесить влияние инструментов при раздельном и совместном применении. Перспективу может слагать метод оценки передачи воздействия, когда применение какого-то инструмента, скажем, монетизации или процентной ставки, влияет не только на денежный или финансовый сектор, но и распространяется далее с какой-то ослабевающей или, наоборот, нарастающей силой по экономике. Для решения такой задачи оценка исходной привязки инструмента к цели политики может играть 
вспомогательную роль, хотя именно эта привязка происходит в ходе стереотипов макроэкономического анализа без идентификации реальной реакции, которая может измениться.

\section{Литература}

1. Абалкин Л. И. Россия: поиск самоопределения. - М.: Наука, 2005. - 464 с.

2. Анчишкин А. И. Прогнозирование темпов и факторов экономического роста. Москва: Макс-Пресс, 2003. - 300 с.

3. Глазьев С.Ю. О путях обеспечения роста российской экономики. Научные труды Вольного экономического общества России. - 2017. - Т. 203. - С. 229-242.

4. Глазьев С.Ю. Битва за лидерство в XXI веке. Россия, США, Китай. Семь вариантов ближайшего будущего. - М.: Книжный мир, 2017. — 352 с.

5. Манделл Р. Выбор экономической политики для достижения внутреннего и внешнего равновесия // Этот изменчивый валютный курс. Сборник статей. - М.: Дело, 2001. - C. 155-164.

6. Манделл Р. Проблема кризисов // Евродитя Манделла? Теория оптимальных валютных зон. Сборник статей. - М.: Дело, 2002. - C. $117-126$.

7. Медоуз Д., Рандерс Й., Медоуз Д. Пределы роста: 30 лет спустя. - М.: Акадкнига, 2007. - $342 \mathrm{c}$.

8. Микульский К.И. Россия в поисках модели экономического роста // Общество и экономика. - 2017. - №3-4. - С. 5-15.

9. Писсаридес К.A. Теория равновесной безработицы. - Издательство СПбГУ, 2018. - 292 c.

10. Сухарев О.С. Инвестиционная политика экономического роста // Вестник ЮжноРоссийского государственного технического университета. Серия: Социально-экономические науки. - 2020 - №2. - С. 7-27.

11. Тинберген Я., Бос X. Математические модели экономического роста. - М.: Прогресс, 1967. - 174 с.

12. Форрестер Дж. Мировая динамика. - М.: ООО «Издательство АСТ», СПб: Terra Fantastica, 2003. - 379 c.

13. Экономика для любознательных: о чем размышляют Нобелевские лауреаты. М.: Институт Гайдара, 2017. — 256 с.
14. Aghion P., Akcigit U., Howitt P. Lessons from Schumpeterian Growth Theory // The American Economic Review. - 2015. Vol. 105. — №. 5. - P. 94-99.

15. Blanchard $O$. The Lucas Critique and Volcker Deflation // American Economic Review. 1984. - Vol. 74 (2), May. - P. 211-215.

16. Hanusch H., Chakraborty L., Khurana $S$. Fiscal Policy Economic Growth and Innovation: An Empirical Analysis of G20 Countries// Levy Economics Institute, Working Paper. - 2017. - №883. - 16 p.

17. Lucas $R$. Econometric policy evaluation a critique // Carnegie-Rochester Conference Series on Public Policy. - 1976. — Vol. 1. - Issue 1. - P. 19-46.

18. Sukharev O.S. Structural analysis of income and risk dynamics in models of economic growth // Quantitative Finance and Economics. - 2020. - Vol. 4 (1). - P. 1-18.

19. Tinbergen J. The Duration of Development // Journal of Evolutionary Economics. 1995. — Vol 5 (3). - P. 333-339.

20. Tinbergen J. Economic Policy: Principles and Design Nortn-Holland. - 1956. - $276 \mathrm{p}$.

21. Welsch H., Kühling J. Macroeconomic performance and institutional change: evidence from subjective well-being data // Journal of Applied Economics. - Vol. 19. - Issue 2. - November, 2016. — Pp. 193-217.

\section{References}

1. Abalkin L.I. Rossija: poisk samoopredelenija [Russia: search for self-determination]. Moscow: Nauka, 2005. - 464 p.

2. Anchishkin A.I. Prognozirovanie tempov i faktorov jekonomicheskogo rosta [Forecasting the rates and factors of economic growth]. Moscow: Maks-Press, 2003. - 300 p.

3. Glaz'ev S. Ju. O putjah obespechenija rosta rossijskoj jekonomiki. Nauchnye trudy Vol'nogo jekonomicheskogo obshhestva Rossii [On ways to ensure the growth of the Russian economy. Scientific Works of the Free Economic Society of Russia]. — 2017. - Vol. 203. - Pp. 229-242.

4. Glaz'ev S. Ju. Bitva za liderstvo v XXI veke. Rossija, SShA, Kitaj. Sem' variantov blizhajshego budushhego [Battle for leadership in the XXI century. Russia, USA, China. Seven variants of the near future]. - Moscow: Knizhnyj mir, 2017. - $352 \mathrm{p}$. 
5. Mandell R. Vybor jekonomicheskoj politiki dlja dostizhenija vnutrennego i vneshnego ravnovesija [The choice of economic policy to achieve internal and external balance] // Jetot izmenchivyj valjutnyj kurs. Sbornik statej [This volatile exchange rate. Collection of articles]. Moscow: Delo, 2001. — Pp. 155-164.

6. Mandell R. Problema krizisov [The problem of crises] // Evroditja Mandella? Teorija optimal'nyh valjutnyh zon. Sbornik statej [Euroditya Mandella? The theory of optimum currency areas. Collection of articles]. - Moscow: Delo, 2002. - Pp. 117-126.

7. Medouz D., Randers J., Medouz D. Predely rosta: 30 let spustja [Limits of growth: 30 years later]. - Moscow: Akadkniga, 2007. $342 \mathrm{p}$.

8. Mikul'skij K.I. Rossija v poiskah modeli jekonomicheskogo rosta [Russia in search of a model of economic growth] // Obshhestvo i jekonomika [Society and Economy]. — 2017. №3-4. - Pp. 5-15.

9. Pissarides K.A. Teorija ravnovesnoj bezraboticy [Theory of equilibrium unemployment]. — Izdatel'stvo SPbGU, 2018. — 292 p.

10. Suharev O.S. Investicionnaja politika jekonomicheskogo rosta // Vestnik Juzhno-Rossijskogo gosudarstvennogo tehnicheskogo universiteta. Serija: Social'no-jekonomicheskie nauki [Investment policy of economic growth. Bulletin of the South Russian State Technical University. Series: Socio-economic sciences]. — 2020 №2. - Pp. 7-27.

11. Tinbergen Ja., Bos H. Matematicheskie modeli jekonomicheskogo rosta [Mathematical models of economic growth]. - Moscow: Progress, 1967. - $174 \mathrm{p}$.

12. Forrester Dzh. Mirovaja dinamika [World dynamics]. — Moscow: OOO
«Izdatel'stvo AST», Saint Petersburg: Terra Fantastica, 2003. - 379 p.

13. Jekonomika dlja ljuboznatel'nyh: o chem razmyshljajut Nobelevskie laureaty [Economics for the curious: what the Nobel laureates think about]. - Moscow: Institut Gajdara, 2017. - $256 \mathrm{p}$.

14. Aghion P., Akcigit U., Howitt P. Lessons from Schumpeterian Growth Theory // The American Economic Review. - 2015. Vol. 105. — №. 5. - P. 94-99.

15. Blanchard $O$. The Lucas Critique and Volcker Deflation // American Economic Review. 1984. - Vol. 74 (2), May. - P. 211-215.

16. Hanusch H., Chakraborty L., Khurana $S$. Fiscal Policy Economic Growth and Innovation: An Empirical Analysis of G20 Countries// Levy Economics Institute, Working Paper. - 2017. — №883. - 16 p.

17. Lucas $R$. Econometric policy evaluation a critique // Carnegie-Rochester Conference Series on Public Policy. — 1976. — Vol. 1. — Issue 1. - P. 19-46.

18. Sukharev O.S. Structural analysis of income and risk dynamics in models of economic growth // Quantitative Finance and Economics. - 2020. - Vol. 4 (1). - P. 1-18.

19. Tinbergen J. The Duration of Development // Journal of Evolutionary Economics. 1995. - Vol 5 (3). - P. 333-339.

20. Tinbergen J. Economic Policy: Principles and Design Nortn-Holland. - 1956. - $276 \mathrm{p}$.

21. Welsch H., Kühling J. Macroeconomic performance and institutional change: evidence from subjective well-being data // Journal of Applied Economics. - Vol. 19. - Issue 2. - November, 2016. - Pp. 193-217. 


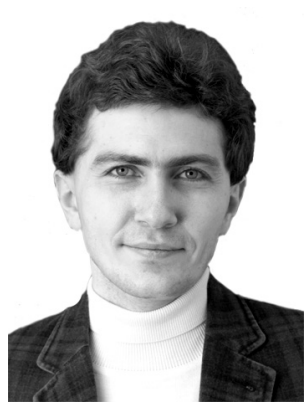

Сухарев Олег Сергеевич - доктор экономических наук, профессор, главный научный сотрудник Института экономики Российской Академии Наук.

Sukharev Oleg Sergeevich - Doctor of Economic Sciense, Professor, Chief Researcher, Institute of Economics, Russian Academy of Sciences.

117218, г. Москва, Нахимовский пр., 32

32 Nakhimovskiy ln., 117218, Moscow, Russia

E-mail: o_sukharev@list.ru 